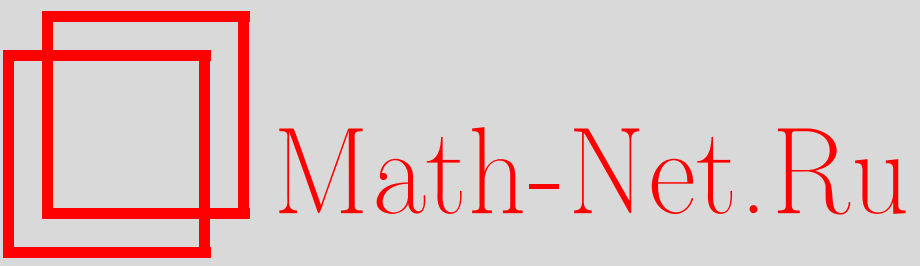

И. О. Золотовский, Д. И. Семенцов, Нелинейная динамика двухмодового волнового пакета в условиях сильной линейной связи, ТМФ, 2003, том 135, номер 1, 107-116

DOI: https://doi.org/10.4213/tmf179

Использование Общероссийского математического портала Math-Net.Ru подразумевает, что вы прочитали и согласны с пользовательским соглашением

http: //www . mathnet.ru/rus/agreement

Параметры загрузки:

IP : 3.85 .183 .62

26 апреля 2023 г., 08:52:21 
ТЕОРЕТИЧЕСКАЯ

И МАТЕМАТИЧЕСКАЯ

ФИЗИКА

Том 135, № 1

апрель, 2003

(C) 2003 г.

И. О. Золотовский* , Д. И. Семенцов*

\title{
НЕЛИНЕЙНАЯ ДИНАМИКА ДВУХМОДОВОГО ВОЛНОВОГО ПАКЕТА В УСЛОВИЯХ СИЛЬНОЙ ЛИНЕЙНОЙ СВЯЗИ
}

\begin{abstract}
Исследуется динамика волнового пакета, формируемого двумя однонаправленными сильновзаимодействующими волнами, распространяющимися в среде с кубической нелинейностью. Для вырожденных ситуаций, когда волновой пакет описывается только одним парциальным импульсом, получено солитонное решение нелинейного уравнения Шредингера. В случае невыполнения условий вырождения с помощью вариационного подхода получено уравнение для длительности импульса, аналогичное уравнению для координаты в кеплеровской задаче. На основе его решения получены зависимости длительности импульса от проходимого расстояния для случаев “финитного" и "инфинитного" режимов распространения.
\end{abstract}

Ключевые слова: волновой пакет, парциальные импульсы, солитонные решения, компрессия.

\section{1. ВВЕДЕНИЕ}

Интерес к структурам с реализуемой сильной линейной связью между однонаправленными волнами определяется разнообразием эффектов, возникаюших при распространении в них световых волновых пакетов, в частности возможностью получения сверхкоротких световых импульсов [1], [2]. К структурам подобного рода относятся туннельно-связанные волоконные световоды (BC), анизотропные и гиротропные среды [3]-[5]. Особый интерес представляют длиннопериодические ВС, для которых период оптической неоднородности по длине световода намного больше длины световой волны [6], [7]. Анализ режимов преобразования оптического излучения в таких ВС выявил сушественную зависимость эффективных дисперсионных параметров от величины межмодовой связи и условий ввода излучения в световод [8], [9]. Последнее обстоятельство дает возможность эффективного управления динамикой излучения в таких системах и реализации в них высоких степеней сжатия импульса, режимов модуляционной неустойчивости, формирования ударных волн и сверхсветовых импульсов [10]-[13]. Данное обстоятельство делает актуальным анализ динамики распространения коротких импульсов по

* Ульяновский государственный университет, Ульяновск, Pоссия. E-mail: SementsovDI@ulsu.ru 
такому световоду с учетом различных нелинейных эффектов. В [14] исследовались особенности распространения коротких импульсов в среде с кубической нелинейностью, реализуемой в стандартных кварцевых световодах, при выполнении условия фазового синхронизма между двумя модами, формирующими импульс, и симметричных типах возбуждения световода, когда поведение всего волнового пакета можно описать только одним парциальным импульсом (ПИ). В настоящей работе исследуется влияние отстройки от фазового синхронизма и несимметричных типов возбуждения на динамику волнового пакета, распространяющегося в периодическом ВС с учетом сильного линейного и нелинейного (кросс-модуляционного) межмодовых взаимодействий и дисперсионных эффектов первого и второго порядков.

\section{2. УРАВНЕНИЯ ДЛЯ МОД И ПАРЦИАЛЬНЫХ ИМПУЛЬСОВ}

Пусть на вход двухмодового световода с периодически меняющимися по длине значениями показателя преломления подается световой импульс длительностью $\tau_{0}$. Эффективная связь меж ду однонаправленными модами, формируюшими импульс, имеет место в области их фазового синхронизма. С учетом периодичности световода и импульсного его возбуждения полный фазовый синхронизм имеет место, если на центральной частоте импульса $\omega_{0}$ обрашается в нуль отстройка от оптического резонанса, т.е. $\delta\left(\omega_{0}\right)=0$, где

$$
\delta(\omega)=\beta_{1}(\omega)-\beta_{2}(\omega)-\frac{2 \pi}{\Lambda}
$$

Здесь $\beta_{j}$ - волновое число $j$-й моды, а $\Lambda$ - период оптической неоднородности световода вдоль оси распространения излучения. В этом случае уравнения для временнь́х огибающих двух взаимодействующих мод, записанные в координатах бегущего времени $\tau=t-z / u$ ( $u$ - групповая скорость волнового пакета), с учетом межмодовой расстройки групповых скоростей, материальной дисперсии и нелинейных эффектов (фазовой самомодулящии и кросс-модуляции) могут быть представлены следующим образом [14]:

$\frac{\partial A_{j}}{\partial z}+\frac{\xi_{j}}{v} \frac{\partial A_{j}}{\partial \tau}-\frac{d_{j}}{2} \frac{\partial^{2} A_{j}}{\partial \tau^{2}}=-i \sigma A_{3-j} e^{-i \xi_{j} \delta z}-i\left(\gamma_{\mathrm{c} j}\left|A_{j}\right|^{2}+\gamma_{\mathrm{k} j}\left|A_{3-j}\right|^{2}\right) A_{j}, \quad j=1,2$.

Здесь введены параметры $\xi_{j}=(-1)^{j}, v^{-1}=\left(u_{1}-u_{2}\right) / 2 u^{2}-$ параметр расстройки обратных групповых скоростей мод, $u_{j}=\left(\partial \beta_{j} / \partial \omega\right)_{0}^{-1}-$ групповая скорость $j$-й моды, $2 u=u_{1}+u_{2} ; d_{j}=\left(\partial \beta_{j}^{2} / \partial \omega^{2}\right)_{0}$ - материальная дисперсия волокна; коэффициент межмодовой связи $\sigma$ и модовые параметры фазовой самомодуляции $\gamma_{c} j$ и кросс-модуляции $\gamma_{\mathrm{k} j}$ определяются соответствуюшими интегралами перекрытия профильных функций волновых мод с учетом распределения оптической неоднородности по сечению и глубины модуляции по длине световода [15]. Следует особо подчеркнуть, что вид уравнений (2) является практически обшим для всех систем с реализуемой сильной линейной связью однонаправленных волн. Так, уравнения, описывающие распространение оптического импульса в туннельно-связанных оптических волноводах, совпадают с (2), но в 
этом случае параметр отстройки определяется лишь разницей модовых констант распространения в волноводах $\delta=\beta_{1}-\beta_{2}$, а параметры $\sigma, \gamma_{\mathrm{c}}, \gamma_{\mathrm{k}}$ определяются несколько отличными от случая периодического волокна соотношениями [5].

Для исследуемого случая сильной межмодовой связи длина межмодового взаимодействия $L_{\sigma}=|\sigma|^{-1}$ должна быть значительно меньше дисперсионной длины $L_{\mathrm{D}}=$ $\tau_{0}^{2} /|d|$, где $d=\left(d_{1}+d_{2}\right) / 2$, длины нелинейности $L_{\mathrm{n}}=\gamma I_{0}$, где $\gamma=\left(\gamma_{\mathrm{c}}+\gamma_{\mathrm{k}}\right) / 2$ и $I_{0}=$ $\left(\left|A_{10}\right|^{2}+\left|A_{20}\right|^{2}\right)$, и длины межмодового разбегания $L_{\mathrm{v}}=v \tau_{0}$. Для легко достижимых значений $|\sigma| \cong 10^{2} \mathrm{M}^{-1}$ и $\gamma \cong 0.1-1\left(\mathrm{~B}_{\mathrm{T}} \cdot \mathrm{M}\right)^{-1}$ использование указанного приближения корректно для интенсивностей вводимого излучения $I_{0} \leqslant 10$ Вт. Поскольку параметр материальной дисперсии $|d| \cong 10^{-26}-10^{-27} \mathrm{c}^{2} / \mathrm{M}$, используемое приближение также справедливо для длительностей вводимого импульса $\tau_{0} \geqslant 10^{-13}$ с.

Уравнения (2) должны решаться совместно с начальными условиями для временнь́х огибающих мод $A_{j}$, определяемыми условиями возбуждения световода. Достаточно общий вид начальных условий представляется соотношением $A_{2}(\tau, 0)=\psi A_{1}(\tau, 0)$, где параметр $\psi$ определяет тип возбуждения волокна. При $\psi= \pm 1$ имеет место симметричное или антисимметричное возбуждение световода, а при $\psi=0$ либо $\psi^{-1}=0$ - одномодовое его возбуждение. С учетом отстройки от фазового синхронизма временна́я огибаюшая соответствуюшей моды может быть представлена в виде комбинации двух ПИ:

$$
\begin{aligned}
& A_{1}=a_{1}(\tau, z) e^{i(q+\delta / 2) z}+a_{2}(\tau, z) e^{-i(q-\delta / 2) z}, \\
& A_{2}=\chi a_{1}(\tau, z) e^{i(q-\delta / 2) z}-\chi^{-1} a_{2}(\tau, z) e^{-i(q+\delta / 2) z} .
\end{aligned}
$$

Здесь $a_{f}$ - медленно меняющиеся с координатой $z$ амплитуды ПИ, параметр $q \equiv\left(\sigma^{2}+\right.$ $\left.\delta^{2} / 4\right)^{1 / 2}$, а определяемый начальными условиями возбуждения световода параметр

$$
\chi=\frac{(2 q+\delta) A_{20}-2 \sigma A_{10}}{(2 q-\delta) A_{10}-2 \sigma A_{20}}=-\frac{(2 q+\delta) A_{10}+2 \sigma A_{20}}{(2 q-\delta) A_{20}+2 \sigma A_{10}} .
$$

При полном фазовом синхронизме $\delta=0$ и параметры $q=|\sigma|$, а $\chi=-1$.

Таким образом, формируемый двумя взаимодействующими модами импульс является суперпозицией ПИ, амплитуды которых в соответствии с (2) и (3) удовлетворяют уравнениям

$$
\frac{\partial a_{f}}{\partial z}-\frac{\xi_{f} \delta}{2 q v} \frac{\partial a_{f}}{\partial \tau}-\frac{i D_{f}}{2} \frac{\partial^{2} a_{f}}{\partial \tau^{2}}+i\left(G_{\mathrm{c} f}\left|a_{f}\right|^{2}+G_{\mathrm{k} f}\left|a_{3-f}\right|^{2}\right) a_{f}=0
$$

Здесь $f=1,2$, параметр $\xi_{f}=(-1)^{f}$, а также введены для соответствующего ПИ эффективные параметры фазовой самомодуляции и кросс-модуляции

$$
\begin{aligned}
G_{\mathrm{c} f}= & \frac{1}{2 q}\left[\left(q+\frac{\xi_{f} \delta}{2}\right)\left(\gamma_{\mathrm{c} 1}+\chi^{-2 \xi_{f}} \gamma_{\mathrm{k} 1}\right)+\left(q-\frac{\xi_{f} \delta}{2}\right)\left(\chi^{-2 \xi_{f}} \gamma_{\mathrm{c} 2}+\gamma_{\mathrm{k} 2}\right)\right], \\
G_{\mathrm{k} f}= & \frac{1}{2 q}\left[\left(2 q+\xi_{f} \delta\right) \gamma_{\mathrm{c} 1}+\left(q+\frac{\xi_{f} \delta}{2}\right)\left(\chi^{2 \xi_{f}}-1\right) \gamma_{\mathrm{k} 1}+\right. \\
& \left.+\left(q\left(\chi^{2 \xi_{f}}+1\right)-\frac{\xi_{f} \delta\left(\chi^{2 \xi_{f}}-1\right)}{2}\right) \gamma_{\mathrm{c} 2}-\xi_{f} \delta \gamma_{\mathrm{k} 2}\right]
\end{aligned}
$$


и эффективной дисперсии

$$
D_{f}=\frac{d_{1}+d_{2}}{2}+\frac{\xi_{f}}{v^{2} q}\left(1-\frac{\delta v^{2}\left(d_{2}-d_{1}\right)}{2}\right) .
$$

Начальные условия для амплитуд ПИ с учетом (4) принимают вид

$$
a_{f}(\tau, 0) \equiv a_{f 0}=\frac{1}{2}\left[A_{10}+\xi_{f}\left(\frac{\delta}{2 q} A_{10}+\frac{\sigma}{q} A_{20}\right)\right]
$$

\section{3. СОЛИТОННЫЕ РЕШЕНИЯ В СЛУЧАЕ ВЫРОЖДЕНИЯ}

Наибольший интерес с точки зрения получения аналитических решений уравнений (4) и их экспериментальной реализации представляет вырожденная ситуация, когда поведение всего волнового пакета можно описать только одним из ПИ. Именно для вырожденной ситуации удается получить оптимальные дисперсионные параметры распространяюшегося в световоде импульса. В случае фазового синхронизма мод $(\delta=0)$ вырождение имеет место при симметричном $(\psi=1)$ либо антисимметричном $(\psi=-1)$ типах возбуждения, при которых $a_{10}=0$ и $a_{20} \neq 0$ либо $a_{20}=0$ и $a_{10} \neq 0$, соответственно. Равенство нулю одной из амплитуд ПИ $a_{f}$ имеет место не только в начальньй момент, но сохраняется в процессе распространения импульса для указанных типов возбуждения световода.

В обшем случае отстройки от фазового синхронизма вырожденная ситуация в соответствии с (7) возможна и для несимметричного типа возбуждения световода. Так, если выполняется условие $\psi_{f}=\left(2 \xi_{f} q-\delta\right) /(2 \sigma)$, то амплитуды ПИ $a_{f} \neq 0, a_{3-f}=0$. При этом система уравнений (4) также вырождается в одно нелинейное уравнение Шредингера [15]:

$$
\frac{\partial a_{f}}{\partial z}-\frac{i D_{f}}{2} \frac{\partial^{2} a_{f}}{\partial \tau_{f}^{2}}+i G_{\mathrm{c} f}\left|a_{f}\right|^{2} a_{f}=0
$$

где введены бегушее время $\tau_{f}=t-z / u_{f}$, связанное с соответствуюшим ПИ, и групповая скорость ПИ $u_{f}^{-1}=u^{-1}-\xi_{f} \delta /(2 q v)$. Полученное уравнение описывает динамику импульса в кубически нелинейной среде с эффективными дисперсией $D_{f}$ и нелинейностью $G_{\mathrm{c} f}$. Характерной чертой описываемого уравнением (8) процесса распространения волнового пакета в такой среде является самовоздействие, приводяшее как к уширению волнового пакета, так и к его сжатию, а также к формированию устойчивых волновых пакетов - шредингеровских солитонов, возникновение которых связано с балансом действия нелинейности среды и дисперсии [15]. В рассматриваемом нами случае сильной межмодовой связи дисперсионные свойства определяются эффективной дисперсией ПИ $D_{f}$, обусловленной материальной дисперсией, межмодовой связью и отстройкой от фазового синхронизма. В случае аномальной эффективной дисперсии $\left(D_{f}<0\right)$ и фокусируюших свойств волноведушей среды по отношению к соответствуюшему ПИ 
$\left(G_{\mathrm{c} f}>0\right)$ уравнение (8) имеет решение, определяюшее так называемые "светлые" солитоны секанс-гиперболической формы. Решение уравнения (8) для амплитуды ПИ в этом случае имеет вид

$$
a_{f}(\tau, z)=a_{f 0} \operatorname{sch}\left(\frac{\tau}{\tau_{\mathbf{i}}}\right) e^{-i \Gamma z}
$$

где фаза, длительность и начальная амплитуда импульса связаны соотношениями $2 \Gamma=$ $G_{\mathrm{c} f} a_{f 0}^{2}=\left|D_{f}\right| / \tau_{\mathrm{i}}^{2}$. Видно, что его длительность сушественным образом связана с параметрами эффективных дисперсии и нелинейности:

$$
\tau_{\mathrm{i}}=\left(\frac{\left|D_{f}\right|}{G_{\mathrm{c} f} a_{f 0}^{2}}\right)^{1 / 2}=\left(\frac{\left|D_{f}\right| \tau_{0}}{G_{f} W_{0}}\right)^{1 / 2}
$$

где $W_{0}=\left(\left|A_{10}\right|^{2}+\left|A_{20}\right|^{2}\right) \tau_{0}$ - плотность вводимой в волокно энергии, а параметр эффективной нелинейности ПИ $G_{f}=\sigma^{2} G_{c f} /\left(q\left(2 q-\xi_{f} \delta\right)\right)$. В случае фазового синхронизма мод $(\delta=0)$ эффективные параметры нелинейности обоих ПИ оказываются одинаковыми и принимают вид $G_{f}=\left(\gamma_{\mathrm{c} 1}+\gamma_{\mathrm{c} 2}+\gamma_{\mathrm{k} 1}+\gamma_{\mathrm{k} 2}\right) / 4$. В случае равенства модовых параметров $\gamma_{\mathrm{c} f}=\gamma_{\mathrm{c}}$ и $\gamma_{\mathrm{k} j}=\gamma_{\mathrm{k}}$ это выражение преобразуется к виду $G_{f}=\left(\gamma_{\mathrm{c}}+\gamma_{\mathrm{k}}\right) / 2$, что соответствует ранее полученным результатам [14]. В случае отстройки от фазового синхронизма $(\delta \neq 0)$ и при равенстве модовых параметров эффективные параметры нелинейности для обоих ПИ также одинаковы и имеют вид

$$
G_{f}=\frac{\gamma_{\mathrm{c}}+\gamma_{\mathrm{k}}}{2}\left(1+\frac{\delta^{2}}{4 \sigma^{2}+\delta^{2}} \frac{\gamma_{\mathrm{c}}-\gamma_{\mathrm{k}}}{\gamma_{\mathrm{c}}+\gamma_{\mathrm{k}}}\right)
$$

Энергия солитоноподобного ПИ для рассматриваемого вырожденного случая с учетом (10) определяется выражением

$$
W_{f}=\frac{W_{0}}{4\left(1+\psi^{2}\right)}\left(1+\xi_{f} \frac{\delta+2 \sigma \psi}{2 q}\right)^{2}
$$

Если плотность вводимой в волокно энергии близка к $W_{f}$, реализуется солитонный режим распространения импульса. При $W_{0}<W_{f}$ импульс расплывается, а при $W_{0}>W_{f}$ импульс сжимается и для степени его самосжатия справедливо аппроксимационное соотношение $\tau_{0} / \tau_{\min } \cong 4 \sqrt{\tau_{0} G_{f} W_{0} /\left|D_{f}\right|}[15]$, где $\tau_{\min }$ - минимальная длительность импульса. Сушественная зависимость эффективных параметров дисперсии и нелинейности от величины отстройки, межмодовой связи и типа возбуждения световода создает условия для эффективного управления степенью компрессии $\tau_{0} / \tau_{\min }$, которая во многом определяет динамику импульса в световоде. 


\section{4. ВАРИАЦИОННЫЙ АНАЛИЗ В ОТСУТСТВИЕ ВЫРОЖДЕНИЯ}

Если рассматриваемая задача не сводится к вырожденным случаям, ее точное аналитическое решение представляется невозможным. В этом случае для ее анализа воспользуемся вариационным методом нахождения приближенного решения, предложенным в работе [16] и успешно применявшимся впоследствии для решения широкого круга задач нелинейной оптики [10]-[12]. В предположении относительной малости отстройки от фазового синхронизма, когда $|\delta / \sigma| \ll 1$, система (4) может быть получена из уравнений Эйлера-Лагранжа в вариационной задаче с лагранжианом $\mathcal{L}=\mathcal{L}_{0}+\mathcal{L}_{w}$. Здесь $\mathcal{L}_{0}-$ невозмушенный лагранжиан, определяемьй соотношением

$$
\begin{aligned}
\mathcal{L}_{0}= & \frac{1}{2} \sum_{f=1}^{2}\left(a_{f} \frac{\partial a_{f}^{*}}{\partial z}-a_{f}^{*} \frac{\partial a_{f}}{\partial z}-\frac{i}{2} D_{f}\left|\frac{\partial a_{f}}{\partial \tau}\right|^{2}-\right. \\
& \left.-i\left(2 G_{\mathrm{c} f}\left|a_{f}\right|^{4}+\left(\gamma_{\mathrm{c} 1}+\gamma_{\mathrm{c} 2}\right)\left|a_{f}\right|^{2}\left|a_{3-f}\right|^{2}\right)\right) .
\end{aligned}
$$

Добавка к лагранжиану $\mathcal{L}_{0}$, учитывающая несимметричность кросс-модуляционных параметров ПИ $\left(G_{\mathrm{k} 1} \neq G_{\mathrm{k} 2}\right)$ в случае отстройки от фазового синхронизма, имеет вид

$$
\mathcal{L}_{w}=\sum_{f=1}^{2}\left(a_{f} F_{f}^{*}+a_{f}^{*} F_{f}\right)
$$

и является малой по сравнению с $\mathcal{L}_{0}$, поэтому может рассматриваться как возмушение. При этом параметры

$$
F_{f}=\left(i\left(\gamma_{\mathrm{c} 1}+\gamma_{\mathrm{c} 2}-G_{\mathrm{k} f}\right)\left|a_{3-f}\right|^{2}-\frac{\xi_{f} \delta}{2 q v} \frac{\partial}{\partial \tau}\right) a_{f}
$$

следует полагать постоянными при вычислении вариационных производных $\delta \mathcal{L}_{w} / \delta a_{f}$ и $\delta \mathcal{L}_{w} / \delta a_{f}^{*}[11],[17]$. Экстремум функционала “действия"

$$
S=\iint \mathcal{L} d z d \tau
$$

достигается на искомых функциях $a_{f}(\tau, z)$ - решениях системы (4). Если выбрать пробные функции $a_{f}\left(\tau, z,\left\{x_{f}\right\}\right)$ зависяшими от набора параметров $\left\{x_{f}\right\}$, вычислить величину $\bar{S}$ на пробных функциях, считая параметры $x_{f}$ зависящими только от $z$ (здесь $\bar{S}=$ $\int \overline{\mathcal{L}}\left\{x_{f}\right\} d z$, где $\left.\overline{\mathcal{L}}\left\{x_{f}\right\}=\int \mathcal{L}\left\{x_{f}\right\} d \tau\right)$, то решения уравнений (4) могут быть найдены из условия $\delta \bar{S}=0$ или $\delta \overline{\mathcal{L}} / \delta x_{f}=0$. В качестве пробного решения, описываюшего амплитуду ПИ, выберем функцию

$$
a_{f}=C_{f} \operatorname{sch}\left(\frac{\theta_{f}}{\tau_{f}}\right) e^{i\left(\varphi_{f}+\alpha_{f} \theta_{f}^{2}\right)}
$$


где введен соответствуюший набор переменных параметров $\left\{x_{f}\right\}: C_{f}(z)$ - амплитуда, $\varphi(z)$ - фаза, $\alpha_{f}(z)$ - скорость частотной модуляшии импульса, $\tau_{f}(z)$ - длительность импульса, $\theta_{f}(z)=\tau-T_{f}(z)$, а параметр $T_{f}$ определяет изменение скорости максимума огибающей импульса. Итак, объектом исследования становится не форма рассматриваемого импульса, а некоторое число основных определяюших его динамику параметров. Достоинством используемого метода анализа является отсутствие необходимости опираться на теорию интегрируемых систем и теорию солитонов.

В силу предполагаемой малости входящих в уравнения (4) членов, характеризующих разбегание ПИ (дисперсионных эффектов первого порядка), припишем каждому из ПИ одинаковые, усредненные по совокупному двухмодовому волновому пакету, значения параметров $\tau_{f} \cong \tau_{\mathrm{i}}, \alpha_{f} \cong \alpha, T_{\mathrm{c} f}=T_{\mathrm{c}}$. Именно указанные параметры в конечном счете определяют динамику ПИ, и для них может быть получена следуюшая система уравнений:

$$
\begin{gathered}
\frac{d\left(\tau_{\mathrm{i}} C_{f}^{2}\right)}{d z}=0, \\
\frac{d \tau_{\mathrm{i}}}{d z}=2 D \alpha \tau_{\mathrm{i}}, \\
\frac{d \alpha}{d z}=\frac{2 D}{\pi^{2}}\left[\frac{1}{\tau_{\mathrm{i}}^{4}}-\pi^{2} \alpha^{2}\right]+2 G\left(\frac{C_{f}}{\pi \tau_{\mathrm{i}}}\right)^{2}, \\
\frac{d T_{\mathrm{c}}}{d z}=\frac{\delta\left(W_{1}-W_{2}\right)}{2 q v\left(W_{1}+W_{2}\right)},
\end{gathered}
$$

где введены эффективные параметры дисперсии и нелинейности волнового пакета

$$
\begin{aligned}
& D=\frac{W_{1} D_{1}+W_{2} D_{2}}{W_{1}+W_{2}}, \\
& G=\frac{G_{\mathrm{c} 1} W_{1}^{2}+G_{\mathrm{c} 2} W_{2}^{2}+\left(G_{\mathrm{k} 1}+G_{\mathrm{k} 2}\right) W_{1} W_{2}}{\left(W_{1}+W_{2}\right)^{2}} .
\end{aligned}
$$

В случае полного фазового синхронизма $(\delta=0)$ выражения для эффективных параметров нелинейности и дисперсии принимают вид

$$
\begin{aligned}
& G=\frac{\gamma_{\mathrm{c} 1}+\gamma_{\mathrm{c} 2}+\gamma_{\mathrm{k} 1}+\gamma_{\mathrm{k} 2}}{2}+\frac{\gamma_{\mathrm{c} 1}+\gamma_{\mathrm{c} 2}-\gamma_{\mathrm{k} 1}-\gamma_{\mathrm{k} 2}}{4}\left(\frac{1-\psi^{2}}{1+\psi^{2}}\right)^{2}, \\
& D=\frac{d_{1}+d_{2}}{2}+\frac{2 \psi}{1+\psi^{2}} \frac{1}{v^{2}|\sigma|} .
\end{aligned}
$$

В соответствии с (17a) $C_{f}^{2} \tau_{\mathrm{i}}=W_{f}=$ const, причем $\tau_{\mathrm{i}}=\tau_{0}$ при $z=0$. Из (17в) и (17г) следует уравнение

$$
\frac{d^{2} \tau_{\mathrm{i}}}{d z^{2}}=p \tau_{\mathrm{i}}^{-2}+Q \tau_{\mathrm{i}}^{-3}
$$


где $p=2 D\left(W_{1}+W_{2}\right) G / \pi^{2}, Q=4 D^{2} / \pi^{2}$. Первый интеграл (20) приводит к уравнению

$$
\left(\frac{d \tau_{\mathrm{i}}}{d z}\right)^{2}=f_{0}-2 p \tau_{\mathrm{i}}^{-1}-Q \tau_{\mathrm{i}}^{-2}
$$

где константа интегрирования $f_{0}=4 D^{2} \tau_{0}^{2} \alpha_{0}^{2}+2 p / \tau_{0}+Q / \tau_{0}^{2}, \alpha_{0}$ - скорость частотной модулящии на входе в световод. Вид уравнения (21) показывает, что решаемая задача сводится к хорошо известному уравнению в задаче Кеплера (см. с.51 в книге [18]). Согласно общей теории этого уравнения характер его решений существенным образом зависит от знака констант $p, Q$ и $f_{0}$. Так, знак параметра $p$ определяет знак "силового центра" и возможность финитного или инфинитного движения, что для импульса в световоде означает конечную длительность (квазисолитонный режим) или уширение его до бесконечности при $z \rightarrow \infty$. Из условия $f_{0}=0$ можно определить суммарную энергию ПИ и, следовательно, значение вводимой в волокно энергии $W_{0}$, превьшение которой приводит к образованию квазисолитонного импульса, т.е. нерасплывающейся уединенной волны. Проанализируем более детально решения уравнения (21), определяющего зависимость длительности импульса $\tau_{\mathrm{i}}$ от пройденного им расстояния при $Q>0$ и $p<0$, когда возможно сжатие импульса.

\section{5. РЕШЕНИЯ И ИХ АНАЛИЗ}

1. $f_{0}<0$. В этом случае уравнение (21) описывает финитное движение или импульс с периодически меняющейся длительностью, который может считаться квазисолитоном (в строгом смысле подобное волновое образование не является солитоном, поскольку исходная система уравнений (2) не является интегрируемой). Решая уравнение (21), для длительности импульса получаем

$$
\tau_{\mathrm{i}}=k(1-\mathrm{e} \cos \zeta)
$$

где параметр Ц связан с пройденным расстоянием соотношением

$$
z=b(\zeta-\mathrm{e} \sin \zeta)+C
$$

Здесь введены обозначения $k=\left|p / f_{0}\right|, \quad b=|p|\left|f_{0}\right|^{-3 / 2}, \mathrm{e}=\left(1-4 \pi^{2}\left|f_{0}\right| /\left(G^{2} W^{2}\right)\right)^{1 / 2}$. Константа интегрирования в (23) определяется из начальных условий и равна $C=$ $b\left(\right.$ e $\left.\sin \zeta_{0}-\zeta_{0}\right)$, а переменная $\zeta$ меняется от $\zeta_{0}$ до $\infty$, где

$$
\zeta_{0} \equiv \zeta(0)=\arccos \frac{k-\tau_{0}}{k \mathrm{e}} .
$$

В рассматриваемом случае параметр е $<1$ и длительность импульса по мере его распространения изменяется в пределах $\tau_{\min } \leqslant \tau_{\mathrm{i}} \leqslant \tau_{\max }$. Минимальная длительность импульса $\tau_{\min }=k(1-\mathrm{e})$ отвечает значениям $\zeta=2 m \pi$ и пройденным во волокну расстояниям $z_{\min }=2 m \pi b+C$, максимальная длительность $\tau_{\max }=k(1+$ е) имеет место при 
$\zeta=(2 m+1) \pi$ в точках $z_{\max }=(2 m+1) \pi b+C$. Если $D \alpha_{0}<0$, то $d \tau_{\mathrm{i}} / d z<0$ и импульс первоначально сжимается до минимальной длительности, после чего наступает фаза уширения. Если же $D \alpha_{0}>0$, то $d \tau_{\mathrm{i}} / d z>0$ и импульс первоначально уширяется до значения $\tau_{\max }$, после чего наступает фаза сжатия импульса. Длина волокна, на которой длительность импульса меняется от максимального до минимального значения, может считаться длиной компрессии для рассматриваемого случая, равной $L_{\mathrm{k}}=\pi b$.

2. $f_{0}>0$. В этом случае уравнение (21) описывает инфинитное движение, т.е. импульс, длительность которого $\tau \rightarrow \infty$ при $z \rightarrow \infty$. В зависимости от знака величины $D \alpha_{0}$ возможны два сценария. Если указанная величина положительна, то импульс непрерывно уширяется начиная с момента его ввода в волокно. Если же $D \alpha_{0}<0$, импульс первоначально сжимается, после чего наступает фаза его уширения. В обоих случаях длительность импульса связана с пройденным расстоянием соотношениями

$$
\tau_{\mathrm{i}}=|k|(\mathrm{e} \operatorname{ch} \zeta-1), \quad z=b(\operatorname{esh} \zeta-\zeta)+C^{\prime}
$$

где $C^{\prime}=b\left(\zeta_{0}-\mathrm{e} \operatorname{sh} \zeta_{0}\right)$, a $\zeta_{0} \equiv \zeta(0)=\ln \left(\varphi+\sqrt{\varphi^{2}-1}\right), \quad \varphi=\left(|k|+\tau_{0}\right) /(|k| \mathrm{e})$. Минимальная длительность импульса и длина компрессии (при $D \alpha_{0}<0$ ) определяются выражениями

$$
\tau_{\min }=|k|(\mathrm{e}-1), \quad L_{\mathrm{k}}=C^{\prime} .
$$

Указанные решения были получены в приближении малости параметра $|\delta / \sigma|$. Как для периодических, так и для туннельно-связанных ВС реализуемые на практике значения параметра $|\delta / \sigma| \leqslant 10^{-3}$. При этом величина $\left|\delta /\left(\sigma v \tau_{0}\right)\right|$, определяющая разбегание ПИ и связанная с дисперсионными эффектами первого порядка, намного меньше обратных длин $L_{\mathrm{D}}^{-1}$ и $L_{\mathrm{n}}^{-1}$. Поэтому для рассматриваемого случая $|\delta / \sigma| \ll 1$ характерно "слипание" отдельных ПИ в единьй волновой пакет. В случае сильной отстройки от фазового синхронизма дисперсионные эффекты первого порядка начинают играть существенную роль. При $|\delta / \sigma| \geqslant 1$ в зависимости от значений длин $L_{\mathrm{v}}, L_{\mathrm{n}}, L_{\mathrm{D}}$ возможны либо полный распад единого волнового пакета на два полностью автономных ПИ, либо сложное по своей динамике взаимодействие ПИ, сопровождаемое периодическим изменением положения их "центров масс". Исследование особенностей динамики ПИ и волнового пакета в целом в этом случае представляет отдельную задачу. Достоверность полученных результатов подтверждается численным решением уравнений (2). Результаты проведенных нами в приближении малости параметра $|\delta / \sigma|$ численных расчетов с исключительно высокой степенью точности совпали с приведенными выше результатами, полученными аналитически с помошью вариационного метода.

\section{6. ЗАКЛЮЧЕНИЕ}

Проведенный анализ показывает, что динамическое поведение двухволнового пакета в волноведущей среде с реализуемой сильной межволновой связью, материальной дисперсией и кубической нелинейностью сходно с поведением одноволнового импульса 
в среде с эффективными параметрами дисперсии $D$ и нелинейности $G$, определяемыми соотношениями (19). При этом в обшем случае отстройки от фазового синхронизма зависимость длительности импульса от пройденного расстояния может описьвать как “финитный”, так и “инфинитный” режимы распространения. Существенное влияние условий ввода излучения на величины эффективных параметров и на их последующую динамику делает перспективным использование подобного рода систем для создания полностью оптических логических элементов.

\section{Список литературы}

[1] В. А. Вислоух, Л. П. Геворкян. Изв. АН СССР. Сер. физич. 1991. Т. 55. № 2. С. 323-328.

[2] J. M. Soto-Crespo, N. N. Akhmediev. Phys. Rev. E. 1993. V. 48. № 6. P. 4710-4715.

[3] А. А. Майер. Квант. электрон. 1982. Т. 2. № 9. С. 1490-1493.

[4] А. Ярив, П. Юx. Оптические волны в кристаллах. М.: Мир, 1987.

[5] A. А. Майep. УФН. 1995. Т. 165. № 9. С. 1037-1075.

[6] С.А. Васильев, Е. М. Дианов, А.С. Курков, О.И. Медведков, В.Н. Протопопов. Квант. электрон. 1997. Т. 24. № 10. С. 151-154.

[7] С.А. Васильев, Е.М. Дианов, Д. С. Стародубов, А.А. Фролов, О.И. Медведков. Квант. электрон. 1997. Т. 24. № 10. С. 160-162.

[8] И. О. Золотовский, Д. И. Семенцов. Опт. и спектр. 1999. Т. 86. № 5. С. 737-739.

[9] И. О. Золотовский, Д. И. Семенцов. Квант. электрон. 1999. Т. 27. № 3. С. 273-277.

[10] А. И. Маймистов. Квант. электрон. 1991. Т. 18. № 6. С. 758-761.

[11] А. И. Маймистов. Квант. электрон. 1994. Т. 21. № 4. С. 358-364.

[12] B. A. Malomed, P.L. Skinner, P.L. Chu, G.D. Peng. Phys. Rev. E. 1996. V. 53. № 4. P. 4084-4091.

[13] И. О. Золотовский, Д. И. Семенцов. Квант. электрон. 2001. Т. 31. № 1. С. 50-54.

[14] И. О. Золотовский, Д. И. Семенцов. Опт. и спектр. 2000. Т. 88. № 4. С. 620-623.

[15] С.А. Ахманов, В.А. Выслоух, А. С. Чиркин. Оптика фемтосекундных лазерных импульсов. М.: Наука, 1988.

[16] D. Anderson. Phys. Rev. A. 1983. V. 27. P. 3135-3141.

[17] D. Anderson, A. Bondenson, M. Lisak. Phys. Lett. A. 1978. V. 67. P. 331-337.

[18] Л. Д. Ландау, Е. М. Лифииц. Механика. М.: Наука, 1988.

Поступила в редакцию 13.XII.2001 г., после доработки 17.IV.2002 г. 